Original Article

\title{
Muscle activity in the lower limbs during push-down movement with a new active-exercise apparatus for the leg
}

\author{
Kenta Tanaka ${ }^{1)}$, Hiroshi Kamada ${ }^{1)^{*}}$, Yukiyo Shimizu ${ }^{1,2)}$, Shizu Aikawa ${ }^{3)}$, Shun Irie ${ }^{4)}$, \\ NaOYuki OChiai ${ }^{5)}$, Masataka SaKane ${ }^{1)}$, Masashi Yamazaki ${ }^{1)}$ \\ 1) Department of Orthopaedic Surgery, Faculty of Medicine, University of Tsukuba: 1-1-1 Tennodai, \\ Tsukuba city, Ibaraki 305-8575, Japan \\ 2) Department of Orthopaedic Surgery, Ibaraki Prefectural University of Health Sciences Hospital, \\ Japan \\ 3) Department of Cardiovascular Surgery, University of Tsukuba Hospital, Japan \\ 4) Department of Integrative Physiology, Graduate School of Medicine, Kyorin University, Japan \\ 5) Department of Orthopaedic Surgery, Kikkoman General Hospital, Japan
}

\begin{abstract}
Purpose] Lower-limb deep vein thrombosis is a complication of orthopedic surgery. A leg-exercise apparatus named "LEX" was developed as a novel active-exercise apparatus for deep vein thrombosis prevention. Muscle activity was evaluated to assess the effectiveness of exercise with LEX in the prevention. [Subjects] Eight healthy volunteers participated in this study. [Methods] Muscle activities were determined through electromyography during exercise with LEX $[\operatorname{LEX}(+)]$ and during active ankle movements [LEX $(-)]$. The end points were peak $\%$ maximum voluntary contraction and \% integrated electromyogram of rectus femoris, vastus lateralis, biceps femoris, tibialis anterior, gastrocnemius, and soleus. [Results] LEX $(+)$ resulted in higher average values in all muscles except the tibialis anterior. Significant differences were noted in the peak of the biceps femoris and gastrocnemius and in the integrated electromyogram of the rectus femoris, vastus lateralis, gastrocnemius, and soleus. The LEX (+)/LEX (-) ratio of the peak was 2.2 for the biceps femoris and 2.0 for the gastrocnemius . The integrated electromyogram was 1.8 for the gastrocnemius, 1.5 for the rectus femoris, 1.4 for the vastus lateralis, and 1.2 for the soleus. [Conclusion] Higher muscle activity was observed with LEX $(+)$. LEX might be a good tool for increasing lower-limb blood flow and deep vein thrombosis prevention.

Key words: Electromyography, Exercise, Venous thrombosis
\end{abstract}

(This article was submitted Oct. 23, 2015, and was accepted Dec. 16, 2015)

\section{INTRODUCTION}

Pulmonary embolism (PE) is a serious, potentially fatal postoperative complication in orthopedic surgery, and lower-limb deep vein thrombosis (DVT) is frequently the source of emboli. Orthopedic surgeries, especially total joint replacement surgery, increase the risk for DVT, and strategies to prevent DVT/PE are important. Venous stasis due to absent muscular contraction is a primary risk factor ${ }^{1)}$. The recommended nonpharmacological methods of prophylaxis, which aim to accelerate venous blood flow, include the use of graduated compression stockings, intermittent pneumatic compression (IPC) devices, and early postoperative mobilization ${ }^{1-3}$.

In particular, active ankle movements and early mobilization have been recommended because prolonged immobility is a primary risk factor for DVT. DVT commonly progresses from the soleus (SOL) vein; therefore, SOL muscle activity is con-

*Corresponding author. Hiroshi Kamada (E-mail: hkamada@tsukuba-seikei.jp)

(C2016 The Society of Physical Therapy Science. Published by IPEC Inc.

This is an open-access article distributed under the terms of the Creative Commons Attribution Non-Commercial No Derivatives (by-nc-nd) License $<$ http://creativecommons.org/licenses/by-nc-nd/4.0/>. 
sidered important, and simple ankle movements are generally recommended ${ }^{1-3)}$. Prior studies have demonstrated increased blood flow and velocity in the femoral vein during active ankle movements in normal subjects, verifying its potential benefits in postoperative patients ${ }^{4,5)}$. However, poor motivation and a lack of skills in the performance of active ankle movements in postoperative patients render their ankle movements ineffective.

Although the effect of calf-muscle activity on regional blood flow is generally considered important ${ }^{6}$, the muscle pump effect is also important for venous blood flow in the lower limb. A leg-exercise apparatus (LEX) was developed as a novel active-exercise apparatus to increase the efficacy of active ankle movements. A previous study showed that the venous flow volume and the maximum and mean venous flow velocities in the femoral vein were higher after 1 min of exercise with active ankle movements with the LEX than those recorded after $10 \mathrm{~min}$ of continuous IPC use ${ }^{7)}$. Furthermore, in a pilot study, the LEX improved the motivation of postoperative patients in performing these movements.

In this study, it was hypothesized that lower-leg muscle activity during exercise with LEX is greater than that with simple active ankle movement, and that this movement with LEX is effective for DVT prevention. However, reports describing leg muscle activity during active ankle movement are lacking.

In this study, the activity of the lower-limb muscles was measured by using surface electromyography (EMG).

\section{SUBJECTS AND METHODS}

The LEX is a portable apparatus consisting of two stainless-steel arms and footplates, with leather soles and straps to secure the feet to the plates (Fig. 1). Structurally, these footplates enable $30^{\circ}$ dorsiflexion, $60^{\circ}$ plantar flexion, $30^{\circ}$ varus, and $20^{\circ}$ valgus, and the arms can revolve around the shaft. The subjects can perform alternate stomping movements. Therefore, stomping of one side can aid the motion of the other side. Furthermore, ankle movement including not only ankle plantardorsiflexion but also varus-valgus movements increases lower-limb venous flow ${ }^{4}$ (Fig. 2). The mechanism does not have specific resistance without friction. LEX has an arch support built into the leather sole, allowing compression of the venous plexus of the plantar of the foot during combined ankle movements (Fig. 3).

Eight healthy volunteers (four men and four women) with a mean age of 26.4 (22-29) years were enrolled in this study. All subjects provided written informed consent, and the study protocol was approved by the ethics committee of Tsukuba University Hospital (approval no. H25-34).

The subjects practiced the combined push-down movement with LEX before the experiment. After skin preparation with sandpaper and alcohol, wireless surface electrodes (WEB- $1000^{\circledR}$; NIHON KOHDEN, Tokyo, Japan) were placed on the center of the muscle belly to assess the EMG activity of the following leg muscles: rectus femoris (RF), vastus lateralis (VL), biceps femoris (BF), tibialis anterior (TA), gastrocnemius (GC), and SOL. Electrodes were placed on the center of the muscle belly, which was identified by means of palpation ${ }^{8)}$.

Exercise 1: The subjects attached the LEX while keeping their knees at $30^{\circ}$ flexion in the supine position, and performed the combined push-down exercise that included not only the ankle but also the knee and hip at $30 \mathrm{cycles} / \mathrm{min}$ for $2 \mathrm{~min}$. This exercise was defined as LEX $(+)$. Exercise 2: The subjects positioned themselves with $30^{\circ}$ knee flexion in the supine position, facilitated by a pillow under their knee, and performed self-managing ankle movements at 30 cycles/min for 2 min, without the LEX. This exercise was defined as active ankle movement: LEX $(-)$.

During these exercises, the subjects were instructed to consciously perform maximum plantar and dorsiflexion that included one cycle of exercise as maximum plantar flexion to the next maximum plantar flexion (Fig. 4).

The sequence of the two exercises was randomized. The subjects rested sufficiently before starting each exercise.

The maximum voluntary contraction (MVC) of each muscle during the isometric exercise was measured by using EMG. EMG measurements were synchronized with a video recording by using a USB camera (DVCap ${ }^{\circledR}$, VitalRecorder $^{\circledR}$; KISSEI COMTEC, Co., Ltd., Nagano, Japan) positioned on the lateral side of subjects.

EMG signals were amplified by a gain factor of 1000 , sampled at $1,000 \mathrm{~Hz}$, and filtered at 30-500 Hz. Ten cycles of EMG with no loss of waves, except for the exercise start and finish, were collected and stored for analysis (BIMTUS-Video ${ }^{\circledR}$, KISSEI COMTEC, Co., Ltd.).

Normalization was conducted with respect to MVC values as $100 \% \mathrm{MVC}^{9}$ ). The peak value was determined from an envelope at $150 \mathrm{~ms}$ smoothing derivative processed zero-phase digital filters of each cycle. Integral EMG (IEMG) was calculated from the total rectified wave of each cycle.

LEX (+) and LEX (-) EMG data (peak IEMG) from all subjects were statistically compared by using a two-way linear mixed model. Post hoc analyses were performed by using the Bonferroni comparisons test. The probability level for statistical significance was $\mathrm{p}<0.05$. Analyses were performed with SPSS (version 21; IBM Corp., Armonk, NY, USA).

This study was supported by the "Japan Science and Technology Agency" grant provided through "A-STEP high-risk challenging type (revitalization promotion type)."

\section{RESULTS}

All subjects completed both exercises without any complication. Movement with LEX resulted in higher average peak and IEMG values in all muscles with the exception of the TA. 
In the thigh muscles, the peak LEX $(+) / \operatorname{LEX}(-)$ ratio was 2.2 for the BF, and the LEX $(+)$ value was significantly higher than the LEX $(-)$ value for the BF (Table 1). In the lower leg, the LEX $(+)$ value was significantly higher than the LEX $(-)$ value for the GC. The peak LEX (+)/LEX (-) ratio was 2.0 for the GC. Although no significant differences were found, the peak LEX $(+)$ value was 1.4 times higher than the LEX $(-)$ for the SOL. However, the LEX $(+)$ value was significantly lower than the LEX $(-)$ value for the TA.

The IEMG values with LEX (+) were 1.5 and 1.4 times higher than with LEX $(-)$ for the RF and VL, respectively, which were significant (Table 2). In the lower-leg muscles, the IEMG value with LEX $(+)$ was 1.8 times higher than the LEX $(-)$ value in the GC and 1.2 times higher in the SOL, which were significant. The IEMG values with LEX $(+)$ in the TA were lower than those with LEX $(-)$; however, this difference was not significant.
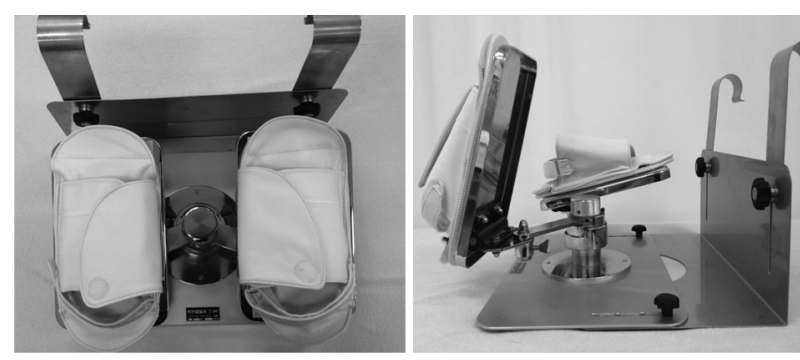

Fig. 1. A novel active-leg-exercise apparatus called LEX

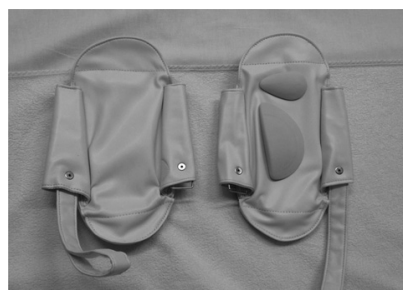

Fig. 3. Soles with arch support pads on the novel activeleg-exercise apparatus called LEX
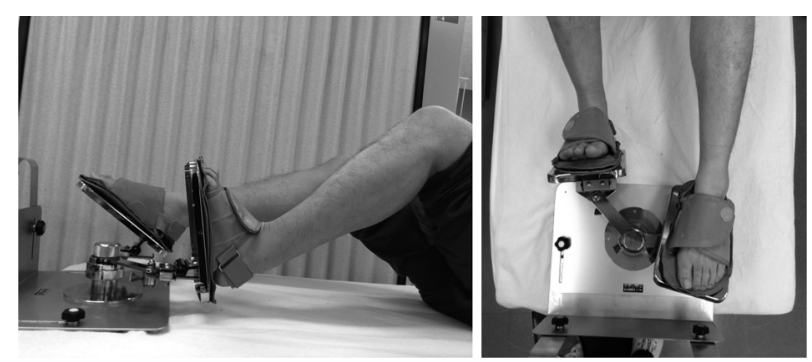

Fig. 2. Lateral (left) and overhead (right) views of the lower-limb movement with the novel active-leg-exercise apparatus called LEX

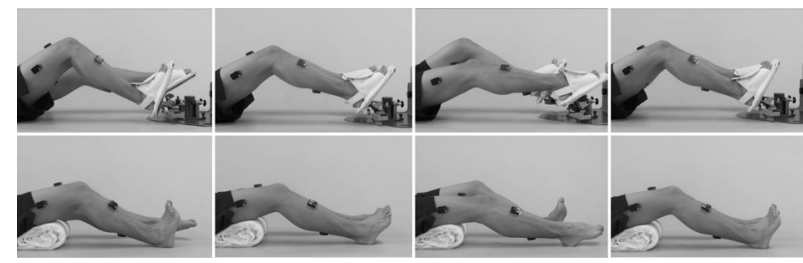

Fig. 4. One cycle of the exercises with the novel active-leg-exercise apparatus called LEX [LEX (+)] (upper) and simple active ankle movements [LEX $(-)]$ (lower)

Table 2. Mean integrated electromyogram (IEMG) values with $\operatorname{LEX}(+)$ and LEX $(-)$

\begin{tabular}{lccc}
\hline \multirow{2}{*}{ IEMG } & $\begin{array}{c}\text { Mea }(+) \\
(\% \mathrm{MVC} \cdot \mathrm{sec})\end{array}$ & $\begin{array}{c}\text { Mean } \\
(\% \mathrm{MVC} \cdot \mathrm{sec})\end{array}$ & $\begin{array}{c}\text { LEX }(+) / \\
\text { LEX }(-)\end{array}$ \\
\cline { 2 - 3 } RF & $3.0 \pm 1.1^{*}$ & $2.0 \pm 0.8$ & 1.5 \\
VL & $3.7 \pm 1.7^{* *}$ & $2.6 \pm 1.4$ & 1.4 \\
BF & $5.2 \pm 3.6$ & $3.8 \pm 3.1$ & 1.4 \\
TA & $5.8 \pm 2.7$ & $6.9 \pm 4.0$ & 0.8 \\
GC & $4.3 \pm 2.5^{* *}$ & $2.4 \pm 1.9$ & 1.8 \\
SOL & $4.2 \pm 2.9^{*}$ & $3.5 \pm 2.5$ & 1.2 \\
\hline
\end{tabular}

LEX (+), exercise using the novel active-leg-exercise apparatus called LEX; LEX (-), simple active ankle movements; RF: rectus femoris; VL: vastus lateralis; BF: biceps femoris; TA: tibialis anterior; GC: gastrocnemius; SOL: soleus

Two-way linear mixed model with Bonferroni post hoc $\mathrm{p}<0.05$ $(*), \mathrm{p}<0.01(* *)$ 


\section{DISCUSSION}

Our hypothesis was that movement with LEX results in higher average values in the GC and SOL. In the present study, of the lower-leg muscles, the GC and SOL had significantly greater muscle activities with LEX than with simple ankle movements. Therefore, it is possible that movements with LEX have a beneficial effect on SOL muscle activity.

The GC works with the SOL for ankle plantar flexion; the relatively large differences between the LEX and the simple ankle movements in the GC were significant. Because a peak value is generally related to muscle contraction, a significant difference in the peak value of GC can be effective in increasing blood flow in the lower leg.

The IEMG in the medial head of the GC reportedly increases with an increase in angular velocity in low-load isotonic exercises, which does not occur in the $\mathrm{SOL}^{10,11)}$. Therefore, the lower level of activity in the SOL, which is mainly an antigravity muscle, might be explained by the primarily low-load dynamic plantar and dorsiflexion movements with LEX ${ }^{12)}$.

The TA activity with LEX was lower than with simple ankle movements. It was inferred that the push-back force by the opposite stomping movement improves ankle dorsiflexion. This result suggests that patients naturally emphasize TA movements when performing simple ankle movements on the bed. Because DVT commonly arises at and progresses from the SOL vein ${ }^{13)}$, patients should emphasize GC and SOL rather than TA movements for DVT prevention. Thus, the LEX might be more effective than simple ankle movements for DVT prevention.

The significant differences in thigh muscle activity were also interesting findings. Kajiyama et al. demonstrated that quadriceps function is more effective than ankle movement in increasing femoral vein blood flow ${ }^{14}$. Movements with LEX result in significantly greater activity of the thigh muscles, which might also increase femoral blood flow.

It can be suggested that the higher activity of the thigh muscles and the emphasis on plantar flexion by using LEX could provide a good effect for increasing venous blood flow.

Concerning study limitations, only healthy subjects were included in this study; therefore, the results might not be generalizable to all patients. Further studies should be conducted with LEX to determine the effect on lower-limb muscle activity in patients. In addition, it is impossible to describe the relation between muscle activity and blood flow on the basis of the present study, and future studies should also be conducted to determine a potential relation.

Movement with the novel leg-exercise apparatus, the LEX, resulted in higher muscle activity in the GC and SOL than with active ankle movements. Therefore, the LEX could be a good tool for mobilization and DVT prevention in patients who are unable to stand up easily.

\section{ACKNOWLEDGEMENTS}

We thank Mr. Toshiji Mizukoshi and Tsutomu Mizukoshi, the executive advisor and executive director at Nemoto Co., Ltd, and the co-producer of LEX, respectively, for their advice on producing the LEX. We would also like to express our gratitude to the late Kiyoshi Eguchi, former professor of the University of Tsukuba Hospital, for his general support and warm encouragement.

\section{REFERENCES}

1) JCS Joint Working Group: Guidelines for the diagnosis, treatment and prevention of pulmonary thromboembolism and deep vein thrombosis (JCS 2009). Circ J, 2011, 75: 1258-1281. [Medline] [CrossRef]

2) Falck-Ytter Y, Francis CW, Johanson NA, et al.: Prevention of VTE in orthopedic surgery patients: antithrombotic therapy and prevention of thrombosis, 9th ed: American College of Chest Physicians Evidence-Based Clinical Practice Guidelines. Chest, 2012, 141: e278S-e325S.

3) Wickham N, Gallus AS, Walters BN, et al. NHMRC VTE Prevention Guideline Adaptation Committee: Prevention of venous thromboembolism in patients admitted to Australian hospitals: summary of National Health and Medical Research Council clinical practice guideline. Intern Med J, 2012, 42: 698-708. [Medline] [CrossRef]

4) Sochart DH, Hardinge K: The relationship of foot and ankle movements to venous return in the lower limb. J Bone Joint Surg Br, 1999, 81: 700-704. [Medline] [CrossRef]

5) McNally MA, Cooke EA, Mollan RA: The effect of active movement of the foot on venous blood flow after total hip replacement. J Bone Joint Surg Am, 1997, 79: 1198-1201. [Medline]

6) Miller BF, Gruben KG, Morgan BJ: Circulatory responses to voluntary and electrically induced muscle contractions in humans. Phys Ther, 2000, 80: 53-60. [Medline]

7) Shimizu Y, Hiroshi K, Tanaka K, et al.: The effect of the ankle active exercise apparatus for improving the venous 
blood flow in the lower extremities. J Jpn Orthop Assoc, 2014, 88: S628.

8) Soda N, Ueki T: Electromyography study of forward-stepping motion. J Phys Ther Sci, 2013, 25: 615-617. [Medline] [CrossRef]

9) Soma M, Murata S, Kai Y, et al.: The activities of the muscles around the ankle joint during foot-gripping are affected by the angle of the ankle. J Phys Ther Sci, 2013, 25: 1625-1627. [Medline] [CrossRef]

10) Tamaki H, Kitada K, Kurata H: Electromyogram patterns during ankle joint movements at various angular velocities in the triceps surae muscles. Jpn J Phys Fit Sports Med, 1993, 42: 292-300.

11) Soma M, Murata S, Kai $Y$, et al.: Kinematic analysis during toe-gripping strength exertion: angular changes in the ankle joint and leg muscle activities. J Phys Ther Sci, 2015, 27: 1957-1960. [Medline] [CrossRef]

12) Tamaki H, Kitada K, Kurata H: Differences in electromyographic behavior of the synergistic muscles during prolonged isometric contractions at different load. Jpn J Physiol Anthropol, 2004, 9: 53-58.

13) Ohgi S, Tachibana M, Ikebuchi M, et al.: Pulmonary embolism in patients with isolated soleal vein thrombosis. Angiology, 1998, 49: 759-764. [Medline] [CrossRef]

14) Kajiyama K, Uno K, Kawamura H: Quadriceps setting is more effective than ankle movement to increase femoral vein blood flow. Hip Jt, 2005, 31: 106-109. 